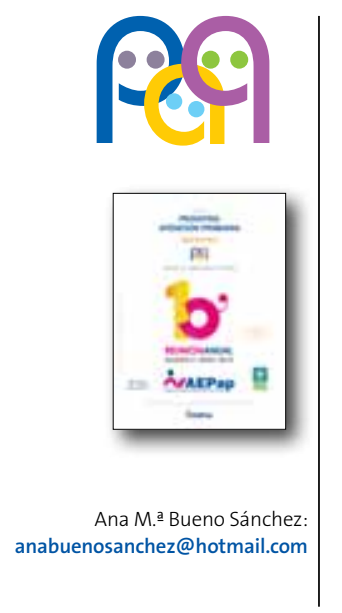

\title{
Exploración de columna y cadera. Cómo manejar la escoliosis
}

\author{
A. M. Bueno Sánchez \\ Hospital Universitario de Getafe, Madrid. España.
}

Desde hace ya unos años, los expertos en raquis consideran la columna vertebral (CV) y su última vértebra, la pelvis, como un todo anatómico funcional y patológico.

De la misma manera, la cadera, articulación de la que forma parte anatómicamente la pelvis, con la aportación por parte de esta del acetábulo óseo, influye de forma fundamental en la fisiopatología de la columna.

Por ello no es descabellado analizar la anatomía y funciones de la CV y de la cadera para poder finalmente comprender la CV patológica.

El repaso de todo esto nos llevará por:

- La cadera:

- Anatomía, funciones y biomecánica.

- Exploración de la cadera sana.

- La CV:

- Anatomía, funciones y biomecánica.

- Exploración de la CV sana.

- Manejo de la escoliosis:

- La actitud escoliótica.

- La escoliosis.

- Hipercifosis dorsal e hiperlordosis lumbar.

\section{LA CADERA}

Por su anatomía, la cadera es poco accesible a la exploración física y la identificación del dolor no es necesariamente en el punto anatómico de la articulación. Ambas cosas favorecen la preocupación del diagnóstico tardío en procesos patológicos de la cadera, pues estos pueden dar lugar a graves secuelas, existiendo como existe una patología muy específica y grave localizada en la misma, aunque, con mucho, la más frecuente sea la más banal.

\section{Anatomía, funciones y biomecánica}

La cadera es una articulación. Pero la articulación es una entidad abstracta, no existe por sí sola: no es la cabeza del fémur, ni es el acetábulo... ni siquiera es la cavidad. La articulación es todo: los extremos óseos con su cartílago articular, la sinovial y la cápsula articular que la envuelve, los ligamentos que la sujetan y las inserciones musculares más o menos tendinosas que la mueven.

Sus funciones son fundamentalmente el sostén del tronco, la transmisión del peso del cuerpo hacia los pies y permitir el movimiento y el desplaza- 
miento del individuo. La cadera se mueve en todos los planos del espacio.

\section{Exploración de la cadera sana}

En la inspección se puede observar la actitud del niño, la posición en la que descansa, y esto es importante. La palpación aporta menos información, aunque puede descartar el origen no articular del proceso. A parte de las maniobras de Barlow y Ortolani, específicas para los primeros días de vida en el diagnóstico de la luxación de cadera, lo fundamental es la valoración del movimiento articular, pasivo y activo, las simetrías del mismo entre las dos caderas y los signos indirectos de ocupación articular, como el signo de Allis-Galleazi.

En el estudio de imagen de la cadera, a parte de las radiografías estándar, es muy importante la ecografía en los primeros meses de vida debido a la ausencia de la osificación de la cabeza femoral. La gammagrafía es una prueba muy usada en la patología del aparato locomotor en el niño, poco específica pero muy sensible. La tomografía computarizada o la resonancia magnética nuclear (RMN) se usan de forma selectiva.

La exploración de la cadera en bipedestación o en la marcha, permite adivinar procesos asociados a una actitud escoliótica (por ejemplo, la fibrosis glútea). La báscula pélvica puede ser causa o consecuencia de una escoliosis. La marcha en trendelemburg podría hacer pensar en una dismetría o en una escoliosis y la luxación congénita bilateral de las caderas, no reducida, producen una hiperlordosis lumbar. En contra, la espondilolistesis lumbosacra también la producen al igual una báscula pélvica hacia anterior.

\section{LA COLUMNA VERTEBRAL}

\section{Anatomía, funciones y biomecánica de la columna vertebral}

A excepción de la cervical, lordosis que no soporta cargas importantes, en la evolución filogenética, se pasa de una columna con una sola curva paralela al suelo a varias curvas perpendiculares al suelo.
Esto supone cambios importantísimos del raquis, que ahora tiene que soporta una gran cantidad de fuerzas axiales que lo comprimen. De hecho, en los homínidos, paso previo en la evolución de la especie, el raquis presenta una sola curva, lo que les obliga a mantener la bipedestación con las rodillas flexionadas y unos brazos largos que sirven de soporte para mantener el equilibrio.

En la ontogénesis humana vemos la misma evolución. En el feto y en los primeros días de vida, la columna lumbar es totalmente cóncava, similar a la de los cuadrúpedos y en el adulto es convexa, consiguiendo enderezar nuestro raquis: al nacer, nuestra CV es una larga cifosis; a los cinco meses, ligeramente cóncava; a los 13 meses, rectilínea; a los tres años aparece la lordosis lumbar, y a los diez años, nuestra CV presenta la curvatura definitiva, prácticamente el raquis del adulto; en el plano lateral estas cuatro curvas son alternas: una lordosis cervical con siete vértebras cervicales, la cifosis dorsal con 12 torácicas, la lordosis lumbar de siete vértebras lumbares y la cifosis sacrocoxígea de cinco vértebras sacras y 3-4 coxígeas.

Las curvas se van compensando para mantener la verticalidad y dar una mayor resistencia al raquis. Con estas cuatro curvas, se consigue diez veces más resistencia.

Conseguida la bipedestación y la marcha bípeda, nuestra CV necesita una anatomía que le permita al individuo la relación con su entorno. Esto se consigue gracias a la resistencia de las vértebras y la elasticidad entre las mismas merced a la interposición de los discos intervertebrales. Esta singular combinación le permite soportar importantes presiones y al mismo tiempo tener una amplia movilidad.

La CV normal está formada por 30 vértebras articuladas entre sí por el disco intervertebral y otras pequeñas articulaciones. Los ligamentos que las unen solidarizan las vértebras haciendo de la CV un todo, el raquis. Por eso, la forma anatómica de las vértebras y los movimientos en cada segmento vertebral es diferente porque el peso que han de soportar es creciente hacia caudal y el fragmento medular que alojan es distinto. 
Los músculos de la columna la mueven y la sostienen. Son músculos muy potentes. A nivel lumbary abdominal, actúan como una faja de múltiples capas entrecruzándose para dar mayor solidez. Otros muchos son delgados, largos e inteligentes que, saltando en ocasiones de vértebra en vértebra, consiguen mover y mantener erguida la CV cual mástil. Y no solo para mantenerla erecta sino también para sostenerla en las distintas posiciones y movimientos que nos permite en conjunto la columna y la cadera: lateralización, rotaciones y flexoextensión.

La CV son 30 huesos, las vértebras. Una unidad funcional y patológica, el raquis. La pelvis sería su última vértebra.

\section{Exploración de la columna vertebral}

\section{En decúbito}

Con en el lactante en prono se puede comprobar la hiperextensión cefálica, el dibujo de la línea de las espinosas, la fuerza de los grupos musculares y la rigidez de los mismos. Con el lactante sentado se ve la línea de las espinosas, si existe o no desviación del tronco y si esta es reductible o si hay gibas.

\section{En bipedestación}

Es muy importante la colocación correcta del niño: siempre descalzo y en ropa interior, recto, estirado, pero flojo, con las piernas en línea separadas el espacio de un pie y bien apoyadas, buscado la posición de "pilates" pero relajado, con el pelo simétricamente colocado, empezando la palpación lejos del tronco para que no se contracture, repitiendo la posición si es necesario. Así se valorará correctamente el espacio braquiotorácico, la escápula prominente, la línea de las espinosas, manchas en la piel, básculas, asimetrías en los pliegues secundarios, etc. En lateral vemos las curvas dorsal y lumbar.

En flexión buscamos el signo de Adams: es la aparición de una giba lateral a la columna. Traduce la existencia de una rotación vertebral y, por ende, de la deformidad costal asociada, ambas solamente presentes si existe una escoliosis estructurada, es decir, una escoliosis. La distancia dedo-suelo y la existencia o no de rigidez de los tendones isquiotibiales son otros aspectos a valorar.

\section{A la marcha}

La exploración nos permitirá recolocar al paciente en bipedestación y valorar cojeras de origen en el requis o en la pelvis, ya mencionadas.

\section{Pruebas de imagen}

Igual que el resto de los huesos del organismo, al nacer la CV no está totalmente osificada. Los arcos posteriores, los pedículos y los platillos vertebrales se osifican con el crecimiento. Los arcos posteriores más tardíos para osificar son los de las vértebras lumbares, que lo hacen en torno a los ocho años, y esto es importante porque se podría confundir con defectos congénitos o lesiones patológicas.

\section{Radiografía simple}

Sigue siendo la exploración más importante. Detecta patología de un tramo de la CV (escoliosis, hipercifosis,) o de una vértebra: infecciones, tumores, lesiones pseudotumorales, lisis-listesis, fracturas, etc. En proyección anteroposterior (AP) y lateral. Las radiografías oblicuas entran en el campo del especialista y, en general, han sido sustituidas por estudios de imagen más profundos.

\section{Telerradiografía}

La proyección AP se usa reiteradamente en el diagnóstico y control evolutivo de la escoliosis.

Es importante identificar la posición escoliótica, diferente de la escoliosis que conlleva una rotación vertebral y que buscaremos en las radiografías. Pero estas deben estar bien posicionadas, incluyendo la pelvis (cuidado con el plomo de protección ovárica).

En proyección frontal y en lateral, la vertical pasa por el mismo punto en la primera vértebra cervical y la última lumbar. 


\section{Otras pruebas}

Otras pruebas son la gammagrafía ósea (hipercaptación en inflamación, infección o tumor). El escáner para el estudio del hueso, útil en las malformaciones congénitas, tumores y pseudotumores, y la RMN, que es excepcional, solo justificable en dudas diagnósticas o en la sospecha de hernia discal.

\section{MANEJO DE LA ESCOLIOSIS}

La CV es una parte del aparato locomotor que no da demasiados problemas. Sin embargo, en las revisiones de salud nos preocupa la posibilidad de no diagnosticar la escoliosis incipiente, que permitirá encauzar su tratamiento a tiempo y evitar terribles deformidades. En este miedo cualquier asimetría de hombros la etiquetamos como escoliosis. No siempre es así... más aún, muy pocas veces es así... La CV puede sufrir distintos procesos patológicos, como un todo, el raquis completo como una unidad funcional, la escoliosis. Pero también puede sufrir procesos que afectan a las vértebras como fragmentos independientes. A su vez, esta lesión aislada en una vértebra puede influir en el todo y manifestarse con una desviación del eje de la misma.

Nuestro objetivo en este apartado es:

- Identificar la escoliosis como tal y diferenciarla de una actitud escoliótica o de una posición escoliótica.

- Pensemos en la dismetría de miembros inferiores como causa de la actitud escoliótica.

- Derivamos a ese paciente al traumatólogo, porque intuimos su patología y en consecuencia lo creemos necesario.

\section{Actitud escoliótica}

La escoliosis es la desviación en el plano AP de la CV (no es recta), pero también hay una rotación (causa de la gibosidad) y una traslación vertebral: es una desviación tridimensional. Si en la exploración física del niño nos parece que un hombro está más bajo que el otro o nos parece que una escápula es más prominente que la otra, no digamos que a lo mejor tiene una escoliosis si no se asocia al resto de los signos y, sobre todo, si el test de Adams es negativo, porque no la tiene. La actitud escoliótica es recuperable.

Para que hablemos de escoliosis, se deben dar las tres desviaciones y esto es lo que la diferencia de la actitud escoliótica, en la cual no hay gibosidad porque no hay rotación vertebral.

Sus causas pueden ser varias. Posicional por posturas inadecuadas: la mochila, el bolso, favorecen la elevación del hombro y producen sobre todo dolor muscular. La dismetría en miembros inferiores. Ocho de cada diez actitudes escolióticas son dismetrías. También pueden ser antiálgicas: tumoral, traumática, infecciosa...

\section{La escoliosis}

En la escoliosis, el signo de Adams es positivo.

La telerradiografía marca la pauta del tratamiento. En ella se valoran un conjunto de aspectos: la curva primaria y la curva secundaria o compensadora. El desequilibrio del raquis, la báscula pélvica, la dismetría. La vértebra límite definida como la primera y la última vértebra que forman parte de la curva. La vértebra clave o apical, que es la vértebra central de la curva y la que presenta mayor rotación. El ángulo de Cobb, definido entre el platillo superior e inferior de las vértebras límite. Este ángulo cuantifica los grados de la escoliosis. El ángulo de Ferguson es menos usado (centro de las vértebras límites). El índice de Moe marca la rotación vertebral y se evalúa por la imagen de los pedículos y las apófisis espinosas en situación central. Varían desde el grado I, en el que se ven los pedículos y las apófisis espinosas, y el grado IV, en el que la apófisis espinosa es lateral y solo se ve uno de los pedículos.

El test de Risser o grado de maduración esquelética, valorado en las crestas ilíacas.

\section{Tipos de escoliosis}

\section{Según su etiología (Scoliosis Research Society, 1966)}

- Idiopática: en el 75-80\% de los casos la causa no es conocida. Es el diagnóstico más frecuente y es más habitual en las niñas, produciendo 
una curva torácica dextro-convexa, con una giba costal en el lado derecho.

- Congénita: este tipo de escoliosis tiene tendencia a progresar desde el nacimiento. Es debida a un defecto de segmentación o de formación de las vértebras.

- Neuromuscular ("paralítica"): causada por la parálisis cerebral, la poliomielitis, la mielitis transversa y las distrofias musculares, la mielodisplasia, la siringomielia...

- Otras causas: conforman un grupo heterogéneo de causas más raras y secundarias a síndromes, tumores vertebrales, fracturas, neurofibromatosis, metabolopatías, displasias óseas, histeria, etc.

\section{Según la edad de aparición}

- Infantil: antes de los tres años. Pueden llegar a ser muy graves.

- Juvenil: entre los cuatro y los nueve años.

- Adolescente: entre los diez años y la madurez esquelética.

Aunque en conjunto se habla de escoliosis precoz y tardía.

\section{Según su localización}

Cervicales. Cervicotorácicas (grave perjuicio estético por desviación de la cabeza). Torácicas, en las que el vértice está entre T2 y T12 y en las que existe un mayor riesgo respiratorio. Toracolumbares y lumbares son las más frecuentes.

\section{Tratamiento}

En general, las curvas entre $10^{\circ}$ y $20^{\circ}$ solo requieren observación clínica y radiológica cada $6 / 12$ meses. Las curvas entre $25^{\circ}$ y $40^{\circ}$ requieren tratamiento ortopédico con corsés y las curvas superiores a $45^{\circ}$ son en principio candidatas a la cirugía.

El tratamiento fisioterápico solo es eficaz en la actitud escoliótica. En la escoliosis solo como complemento a los otros tratamientos; ayuda a la potenciación muscular y a flexibilizar las curvas.
El tratamiento ortopédico con yesos aplicados bajo anestesia general es muy poco habitual, y está indicado solo en la rara escoliosis infantil progresiva. En el resto de los casos, el tratamiento con corsés se aplicará durante el crecimiento, especialmente en su fase acelerada (signo de Risser $0, \mathrm{I}, \mathrm{II}$ ), en el caso de pacientes femeninas, antes de la menarquia o como máximo durante el año siguiente a la misma, y solo en curvas entre $25^{\circ}$ y $40^{\circ}$. Los corsés no suelen corregir las curvas pero sí pueden frenar su progresión. Se considera un éxito del tratamiento ortopédico cuando las escoliosis progresan $5^{\circ}$, siempre que no requieran tratamiento quirúrgico una vez finalizado el crecimiento. Alrededor de un $30 \%$ de las escoliosis tratadas ortopédicamente evolucionan a pesar del tratamiento ortopédico. Sin embargo, los criterios de tratamiento con corsé, o el tipo de corsé, o las horas de aplicación, no están nada claros y ni los más expertos en raquis se ponen de acuerdo.

En general, los factores de progresión son:

- La edad: en general, las curvas evolucionan en periodos de crecimiento rápido. En el estirón puberal la evolución es muy rápida. Un test de Risser 0 ol.

- El patrón de la curva: curvas dobles, particularmente las torácicas dobles (ápex en T12). Las lumbares son las de menor riesgo.

- Grados de la curva: las curvas torácicas de $30^{\circ}$.

- El sexo: en las niñas es diez veces mayor el riesgo de progresión.

\section{Criterios de derivación}

Lo más importante es distinguir una actitud escoliótica, que no hay que derivar, de una escoliosis.

Pero cuando en la exploración sistematizada de la CV observamos un aspecto físico compatible con escoliosis, la mayoría de los traumatólogos infantiles y de raquis creemos que ya existe criterio de derivación, con telerradiografías hechas, que en la primera ocasión deben hacerse en las dos proyecciones, AP y lateral. Solo las curvas de menor importancia, de $10^{\circ}$ a $20^{\circ}$ y cuya evolución no progresa más de $2^{\circ}$ cada seis meses, podrían ser 
controladas por el pediatra, pero en general deben ser derivadas, máxime si existen criterios de progresión.

\section{HIPERCIFOSIS DORSAL E HIPERLORDOSIS LUMBAR}

La cifosis, definida en la región dorsal, es normalmente de $30^{\circ}$ a $40^{\circ}$. La hipercifosis puede ser debida a muchas causas:

- Fisiológica: durante la primera infancia, muchos niños presentan una cifosis cervicodorsal fisiológica y un genu recurvatun como forma de mantener el centro de gravedad en su sitio.

- Posicional o del adolescente: la niña que trata de ocultarse o la adolescente lánguida. Con la edad cambia completamente esta actitud y lo corrigen espontáneamente.

- Osteocondrosis juvenil o enfermedad de Scheuermann: los platillos vertebrales de las vértebras torácicas de estos adolescentes presentan un acuñamiento anterior. Los pacientes suelen quejarse de dolor lumbar transitorio y no invalidante debido a la lordosis lumbar compensadora. Cuando el Scheuermann es lumbar, lo que ocasiona es una disminución de la lordosis lumbar.

- Infecciones: es poco frecuente esta patología como causa de cifosis en el niño. Pero no es excepcional la tuberculosa, que produce una angulación pronunciada (giba central) y puede producir compresión de la médula espinal y parálisis.

- Metabolopatías, displasias ósea, tumoral, congénita, traumática y otras.
En la escoliosis idiopática, la columna torácica presenta una pérdida relativa de la cifosis normal, se llama hipocifosis o "dorso plano".

La hiperlordosis lumbar casi siempre es una deformidad compensadora de una deformidad primaria en cifosis en cualquier segmento de la CV. También es la respuesta lógica a las deformidades fijas en flexión a nivel de la cadera:

- La hiperlordosis fisiológica es muy frecuente durante la primera infancia como mecanismo compensador de una hipercifosis dorsal fisiológica. Se llama lordosis juvenil benigna.

- Posicional. En el embarazo. Los tacones altos.

- Displasia óseas, tumoral, congénita, traumática.

- La espondilólisis con espondilolistesis.

La evolución y el tratamiento de ambas dependen de la causa que las provoca. Las más frecuentes, las fisiológicas y las posicionales, se corrigen con cinesiterapia y autocorrección.

La eficacia de los corsés en la cifosis es más que incierta y solamente en aquellas de grados próximos a 80 puede estar indicado el tratamiento quirúrgico.

\section{CONFLICTO DE INTERESES}

Los autores declaran no presentar conflictos de intereses en relación con la preparación y publicación de este artículo.

\section{ABREVIATURAS}

AP: anteroposterior $\bullet \mathrm{CV}$ : columna vertebral $\bullet$ RMN: resonancia magnética nuclear. 


\section{BIBLIOGRAFİA RECOMENDADA}

- Dayer R, Haumont T, Belaieff W, Lascombes P. Idiopathic scoliosis: etiological concepts and hypotheses. J Child Orthop. 2013;7:11-6.

- Konieczny MR, Senyurt H, Krauspe R. Epidemiology of adolescent idiopathic scoliosis. J Child Orthop. 2013;7:3-9.

- Padulo J, Ardigò LP. Vertebral rotation in adolescent idiopathic scoliosis calculated by radiograph and back surface analysis-based methods: correlation between the Raimondi method and rasterstereogra- phy. Eur Spine J;22:2336-2337: Statistical perspectives part II. Eur Spine J. 2014;23:922-3.

- Pasha S, Aubin CE, Parent S, Labelle H, Mac-Thiong JM. Biomechanical loading of the sacrum in adolescent idiopathic scoliosis. Clin Biomech (Bristol, Avon). 2014;29:296-303.

- Pourfeizi HH, Tabrizi A, Bazavar M, Sales JG. Clinical findings and results of surgical resection of thoracolumbar osteoid osteoma. Asian Spine J. 2014;8:150-5.

- Wang MY. Miniopen Pedicle Subtraction Osteotomy: Surgical Technique and Initial Results. Neurosurg Clin N Am. 2014;25:347-51. 